\title{
Electron irradiance of conductive sidewalls: A determining factor for pattern-dependent charging
}

\author{
Gyeong S. Hwang and Konstantinos P. Giapis ${ }^{\text {a) }}$ \\ Division of Chemistry and Chemical Engineering, California Institute of Technology, Pasadena, \\ California 91125
}

(Received 7 March 1997; accepted 7 July 1997)

\begin{abstract}
Numerical simulations of charging and profile evolution during gate electrode overetching in high density plasmas have been performed to investigate the effect of long conductive sidewalls on profile distortion (notching). The results reveal that the angle of electron irradiance of the conductive portion of the sidewalls affects profoundly the charging potential of the gates. Larger angles, obtained for thinner masks and/or thicker polysilicon, result in reduced gate potentials which, through their influence on the local ion dynamics, cause more severe notching at all lines of the microstructure. (C) 1997 American Vacuum Society. [S0734-211X(97)02805-9]
\end{abstract}

\section{INTRODUCTION}

Charging damage, occurring during gate electrode overetching of metal-oxide-semiconductor devices in highdensity plasmas, is manifested in at least two ways: (a) sidewall profile distortion (notching), ${ }^{1}$ originating from the deflection of ions near the trench bottom-caused by significant charging of the cleared oxide, ${ }^{2}$ and (b) gate oxide degradation by tunneling currents driven by the charging potential of the gate electrodes. ${ }^{3}$

Charging damage is influenced by the aspect ratio (etch depth over trench width). ${ }^{4}$ For a given plasma, the charging potential at the bottom of each trench reflects the balance between the electron and ion currents flowing to the bottom insulating surface. ${ }^{2}$ Since the electron angular distribution at the wafer is more isotropic than the ion angular distribution, ${ }^{5}$ the aspect ratio determines the degree of electron shadowing of the trench bottom and, thus, the electron flux to that surface. The highly directional ion flux is not significantly affected by the aspect ratio. Therefore, increasing the aspect ratio decreases the electron current to the bottom surface; the ion current to the same surface must also be reduced accordingly to reach steady state. The reduction is achieved by the buildup of a larger potential at the bottom surface, forcing more ions to be deflected. A larger ion flux to the poly-Si sidewalls causes the corresponding line potentials to increase. Thus, an increasing aspect ratio is expected to worsen both forms of charging damage. ${ }^{4}$

As device rules get smaller, aspect ratios tend to increase. Combined with a reduction in gate oxide thickness-for larger transistor current and control of short-channel effects-increased gate electrode potentials during plasma etching could be detrimental to the device. Furthermore, profile peculiarities cannot be tolerated at the smaller linewidths. Profile distortion is a result of sidewall etching by the deflected ions. Since these ions lose a significant portion of their translational energy in the deflection process, the overetch chemistry may be modified so that the threshold for

\footnotetext{
a) Author to whom correspondence should be addressed; Electronic mail: giapis@cheme.caltech.edu
}

reaction is increased, ${ }^{2}$ thus preventing the lower energy ions from reacting. For example, addition of oxygen during overetching is a widely practiced chemical solution to the notching effect. ${ }^{6}$ Silicon oxychlorides are more difficult to remove than $\mathrm{SiCl}_{x} ;{ }^{7}$ thus, sidewall etching is reduced while the trench bottom continues to etch by higher energy (undeflected) ions.

Unless a thick insulating layer deposits at the sidewalls, a change in the etch chemistry does not influence the charging potential of the corresponding gate electrodes. As long as these potentials remain large, the problem of gate oxide degradation will persist. One way to reduce the gate electrode potentials consists in allowing more plasma electrons to reach their sidewalls. ${ }^{8}$ This is the case for the edge line of the pattern, which receives more electrons from the side facing the open area. ${ }^{4}$ When not electrically connected to the edge line, the intermediate lines do not afford the same luxury; plasma electrons can reach their conductive sidewalls only through the trench opening. Thus, the geometric angle $\phi$ of electron irradiance of the conductive part of the sidewalls plays a critical role in allowing the necessary electrons to reach the gate electrode and reduce its charging potential. For a fixed aspect ratio, $\phi$ is increased by increasing the gate electrode thickness or, equivalently, by decreasing the mask thickness. We have demonstrated elsewhere ${ }^{8}$ the dramatic reduction in the charging potentials accompanying the decrease in mask thickness. However, the numerical results of that study are difficult to realize in practice where, among other problems, selectivity requirements must be met. One possible solution to the selectivity issue is to switch from a photoresist mask (thick) to a hard oxide mask (thin), with a concomitant reduction in the aspect ratio. Indeed, such a substitution would increase the electron irradiance of the gate electrode $(\phi \uparrow)$ yielding the desired result. For a fixed mask thickness and a given device rule, $\phi$ can also be increased by independent manipulation of the gate electrode thickness. ${ }^{9}$ The effect of the resulting increase in the aspect ratio on both forms of charging damage is the subject of the present article. We seek to distinguish between the effect of the increase in aspect ratio by increasing the gate electrode thick- 

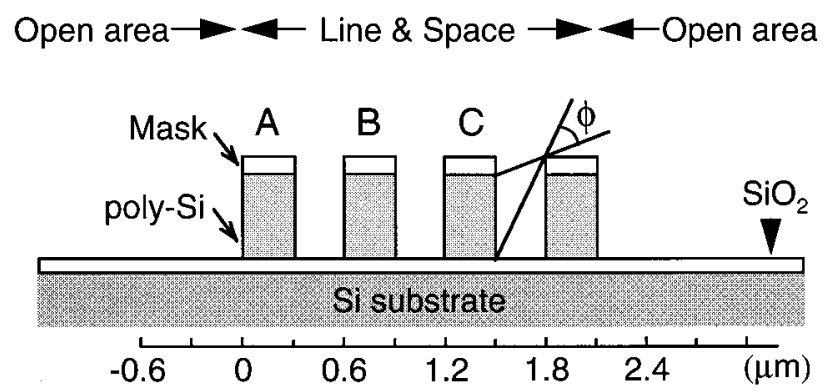

FIG. 1. Schematic depiction of the line-and-space structure considered in the simulation. The polysilicon thickness is varied from 0.2 to $1.1 \mu \mathrm{m}$ while the mask thickness remains constant at $0.1 \mu \mathrm{m}$. The open areas are assumed to be infinite. Note the definition of the angle $\phi$ of electron irradiance of the poly-Si sidewall through a trench opening.

ness $($ mask thickness $=$ constant $)$ versus that produced by increasing the mask thickness (gate electrode thickness $=$ constant). The two effects are different because $\phi$ increases in the former while it decreases in the latter case.

Charging and sidewall profile evolution are performed as described in detail elsewhere. ${ }^{2}$ The methodology and procedures will not be outlined here; a synopsis has appeared in two additional publications, ${ }^{4,8}$ addressing complementary effects.

\section{PLASMA CONDITIONS AND MICROSTRUCTURE DESCRIPTION}

We simulate a low pressure $(<10$ mTorr $)$ chlorine plasma with a density of $1 \times 10^{12} \mathrm{~cm}^{-3}$. The wafer electrode is biased; the sheath voltage is given by $37+30 \sin \omega t \mathrm{~V}$, where $\omega=400 \mathrm{kHz}$ is the rf bias frequency. The ion and electron temperatures are assumed to be 0.5 and $4.0 \mathrm{eV}$, respectively. The chlorine is assumed to be $100 \%$ dissociated at the relatively high plasma density considered. ${ }^{10}$

The dense structure of Fig. 1 borders widely open areas and consists of $0.3 \mu \mathrm{m}$ lines separated by $0.3 \mu \mathrm{m}$ spaces (trenches); the lines are not electrically connected. At the onset of overetching, each feature consists of a hard mask $\left(\mathrm{SiO}_{2}\right)$ onto poly-Si; the features are formed on top of a uniform layer of $\mathrm{SiO}_{2}$, thick enough $(>100 \mathrm{~nm})$ to prevent tunneling currents to the Si substrate. ${ }^{11}$ The hard mask is 0.1 $\mu \mathrm{m}$ thick (constant) while the poly-Si thickness will be varied from 0.2 to $1.1 \mu \mathrm{m}$. The poly-Si is assumed to be heavily doped (electrically conductive).

\section{MICROSTRUCTURE CHARGING RESULTS}

We begin by plotting the steady-state charging potential distribution in both the "edge" trench (between features A and B) and the "middle" trench (between features B and C), shown in Figs. 2 and 3 for various poly-Si thicknesses $\left(t_{p}\right)$. These plots reveal the perturbation in the local ion dynamics that accompanies the enlargement of the poly-Si sidewalls by illustrating the potential felt by the ions as they traverse the trench region confined by the poly-Si sidewalls [depicted at the inset of Fig. 2(a)]. Gradients on this potential surface are a measure of the electric field that influences ion motion. ${ }^{2}$ The bimodal potential distribution at the trench bottom, obtained for $t_{p}=0.4 \mu \mathrm{m}$ [Fig. 2(a)], with sharp peaks near the poly-Si sidewalls, forecasts notch formation at both sidewalls. ${ }^{2}$ Since the peaks are not symmetric, the notches will not be identical; the more pronounced peak by the inner side of the edge line will cause the formation of a deeper and broader notch. On the contrary, the bimodal potential distribution in the middle trench, shown in Fig. 3(a), suggests the formation of symmetric notches at both sidewalls of that trench. Notice that each of these peaks is intermediate in height and width between the two peaks of the potential distribution in the edge trench. As discussed elsewhere, ${ }^{12}$ these differences lead to a peculiar dependence of the notch depth and shape on the feature location along the line-andspace pattern.

As the poly-Si thickness is increased to $t_{p}=0.6 \mu \mathrm{m}$ [Fig. 2(b)], both peaks of the potential distribution get larger and broaden; each maximum shifts towards the center of the trench. The same is true for the potential distribution in the middle trench [Fig. 3(b)]. The trend is better illustrated for $t_{p}=1.1 \mu \mathrm{m}$ [Figs. 2(c) and 3(c)]. In the latter two cases, more incoming ions-those that were approaching the bottom surface nearer to the trench centerline-will encounter a larger repulsive potential and are expected to undergo deflection towards the sidewalls. Since the potential maxima grow larger, the average energy of the deflected ions should also increase. The combined observations suggest worsening of notching at all lines.

The expected reduction in the magnitude of the calculated charging potential of the neighboring poly-Si line (B) with increasing poly-Si thickness is demonstrated in Fig. 4. Surprisingly, the potential of the edge poly-Si line (A) actually increases! First, note that the absolute potential values of both lines are significantly lower than those in features with comparable aspect ratio but shorter poly-Si sidewalls-and, thus, thicker insulating mask. ${ }^{4}$ The thin mask $(0.1 \mu \mathrm{m})$ allows larger electron irradiance of the poly-Si sidewalls and lower charging potentials. Second, increasing poly-Si thickness increases $\phi$, which explains the further drop in the potential of line B. Since the same is true for line A, why does its potential increase? The charging potential of each line reflects the initial imbalance of electron and ion currents. As the sidewalls get thicker, both the electron and ion flux to line A increase, the former due to increased electron irradiation of both sidewalls while the latter due to the perturbation in the in-trench ion dynamics ${ }^{13}$ (see Fig. 2). The physics is such that the increase of the ion flux exceeds that of the electron flux. Thus, the potential of line A must increase until it can attract more electrons from the side facing the open area to balance the total ion current. On the contrary, the increase in the ion flux to the sidewalls of line B is smaller than the increase in the electron flux to the same sidewalls, occurring at the larger electron irradiance angle. Third, the trend for line B as a function of the aspect ratio is exactly the opposite from that seen for the same line, when the mask thickness was varied at constant poly-Si thickness. ${ }^{4}$ 

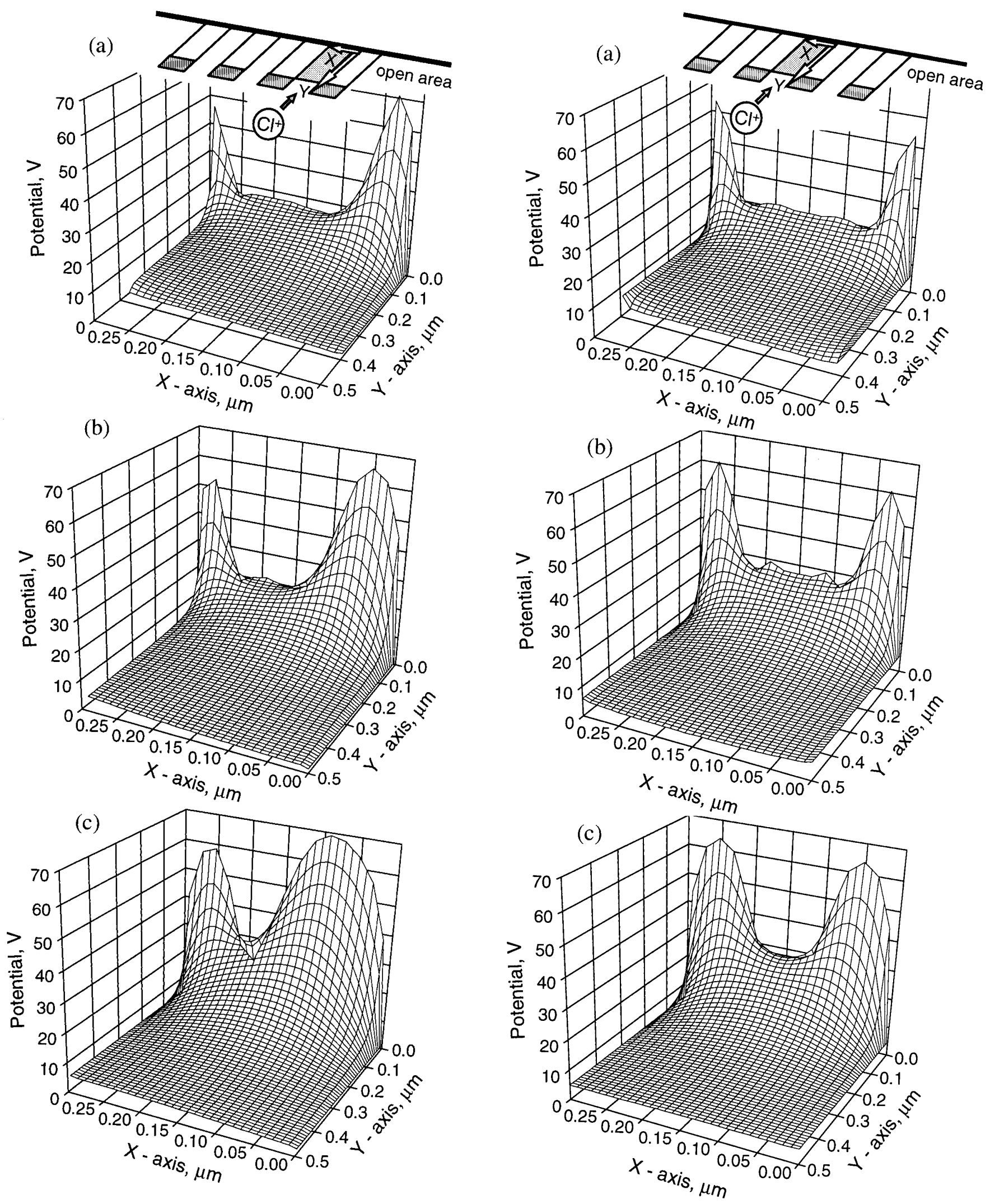

FIG. 2. Three-dimensional charging potential distributions in the edge trench for structures with a polysilicon thickness of: (a) 0.4, (b) 0.6, and (c) 1.1 $\mu \mathrm{m}$. The inset in (a) illustrates the area of interest (extending $0.5 \mu \mathrm{m}$ from the trench bottom) and defines the origin for the potential surface. The microstructure has been rotated so that the direction of ions as they approach the potential surface corresponds to the ion motion in the trench (arrow).

FIG. 3. Three-dimensional charging potential distributions in the middle trench for structures with a polysilicon thickness of: (a) 0.4, (b) 0.6, and (c) $1.1 \mu \mathrm{m}$. The inset in (a) illustrates the area of interest (note difference with Fig. 2). 


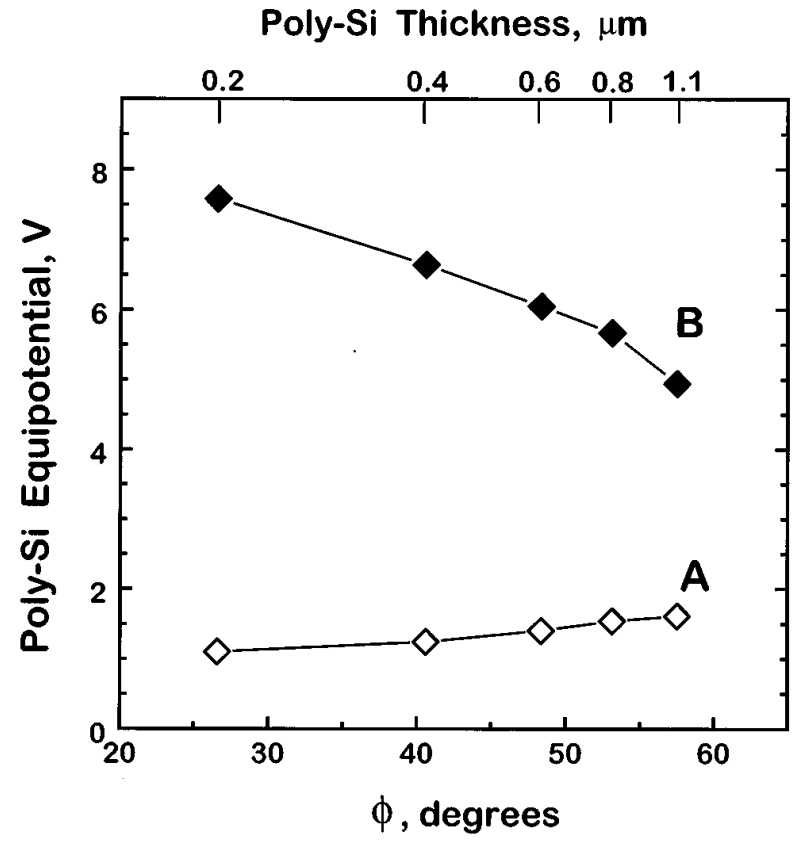

FIG. 4. The steady-state equipotentials of the poly-Si edge line (A) and its neighboring line (B), as a function of the angle $\phi$ of electron irradiance of the poly-Si sidewall. For definitions, see Fig. 1.

If the results of that study were plotted as a function of the angle of electron irradiance $\phi$, the trend would have been identical: the equipotential of intermediate poly-Si lines decreases with increasing $\phi$. This observation implies that data relating to charging damage should be plotted against $\phi$ rather than aspect ratio.

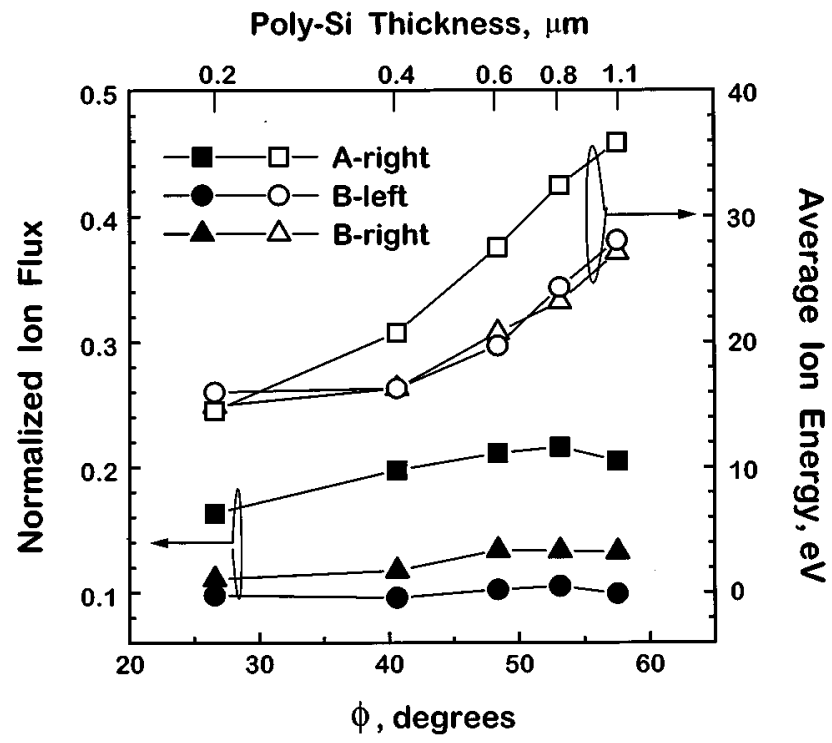

FIG. 5. The normalized flux of ions arriving at the trench bottom and at the first $0.2 \mu \mathrm{m}$ (from the poly-Si/ $\mathrm{SiO}_{2}$ interface) of the inner poly-Si sidewall of the edge line (A-right) and both sidewalls of its neighboring line (B-left, B-right), after steady-state charging has been reached, as a function of the electron irradiance angle $\phi$. The average incident energy of the ions impinging at the corresponding sidewalls (first $0.2 \mu \mathrm{m}$ ) is also shown. For definitions, see Fig. 1.

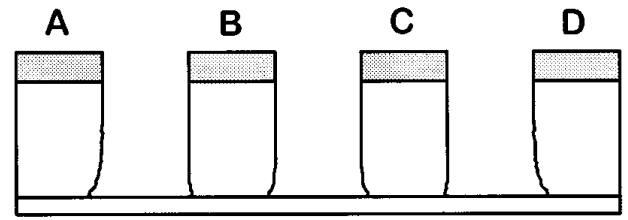

(a)

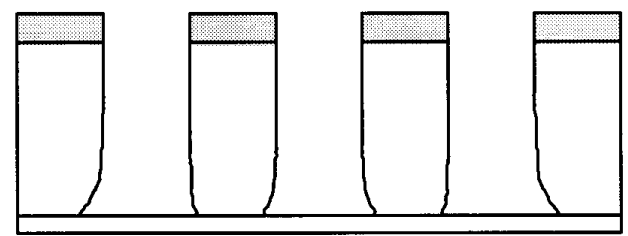

(b)

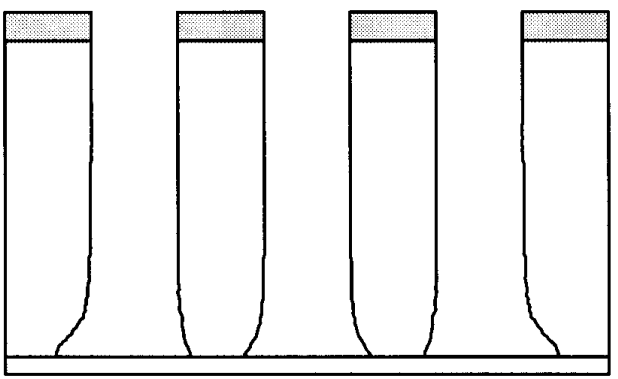

(c)

FIG. 6. Notch profiles predicted for fixed (arbitrary) overetching time of structures with a polysilicon thickness of: (a) 0.4, (b) 0.6 , and (c) $1.1 \mu \mathrm{m}$. The shaded rectangles are the mask, the profiled area underneath is the poly-Si, and the thin rectangle below represents the $\mathrm{SiO}_{2}$. The aspect ratio has been preserved. The profile evolution of the outer sidewall of the edge lines is not simulated.

The decrease in the charging potential of the neighboring line, combined with the (still) low potential of the edge line, suggests that thinner gate oxides can be used without fear for catastrophic leakage currents. Indeed, the neighboring poly-Si line potential drops to $5 \mathrm{~V}$ for the tallest structure studied. Such a low voltage could be tolerated by extremely thin $(4-6 \mathrm{~nm})$ gate oxides. ${ }^{14}$ Thus, use of a thinner mask (e.g., $0.1 \mu \mathrm{m}$ ) seems promising for a reduction of gate oxide degradation, even for structures with an aspect ratio as high as 4:1. Although this prediction suggests an interesting experiment for the $0.3 \mu \mathrm{m}$ device rule, its full potential should be realized at smaller device rules $(\leqslant 0.18 \mu \mathrm{m})$, where the use of thinner masks could be proven indispensable for alleviating charging problems.

The perturbation of the ion dynamics in the trench is quantified in Fig. 5, where the normalized ion fluxes to the trench bottom and the poly-Si sidewalls are plotted as a function of $\phi$. To make the comparison of ion fluxes meaningful, only the flux to the first $0.2 \mu \mathrm{m}$ from the poly-Si/ $\mathrm{SiO}_{2}$ interface-comprising the notch area-is calculated. The flux of deflected ions to the edge poly-Si line (A-right) increases with $\phi$, peaks at $\phi=53^{\circ}$ and then drops. There is 


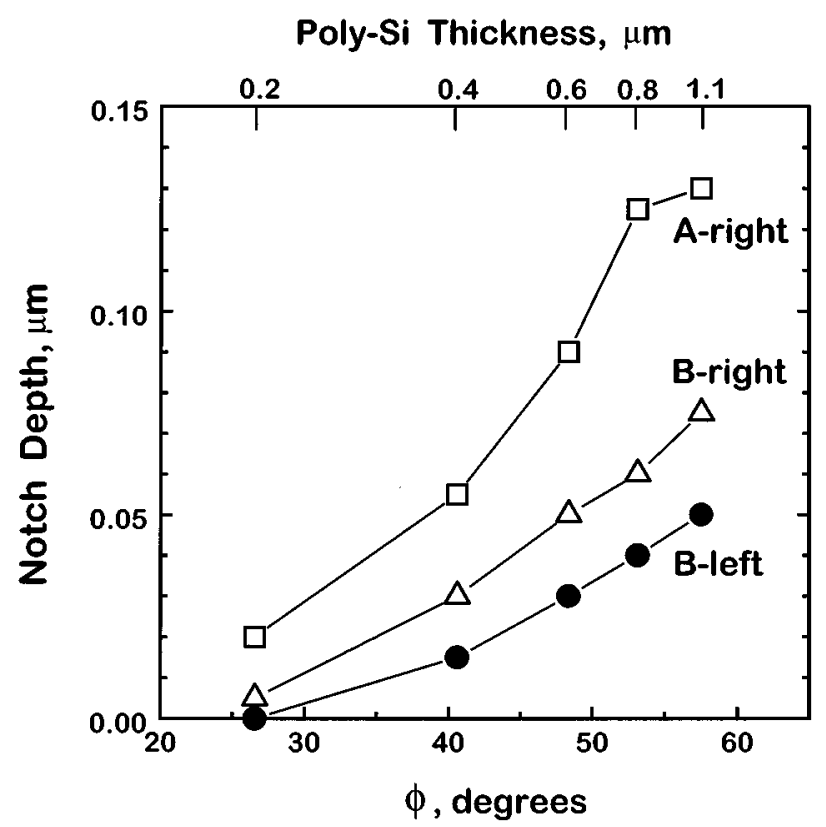

FIG. 7. Dependence of the notch depth at various sidewalls on the electron irradiance angle $\phi$. For definitions, see Fig. 1.

also a slight increase in the ion fluxes to the sidewalls of the neighboring line (B-right, B-left). The increase in the ion fluxes to the sidewalls occurs at the cost of the ion flux to the trench bottom (not shown). On the same graph (right axis), we also show the average ion energy to the first $0.2 \mu \mathrm{m}$ of the sidewalls; all curves increase monotonically with $\phi$. These trends are consistent with the change in the potential distribution at the trench bottom (see Figs. 2 and 3). Since etching of the sidewalls depends on both the energy and flux of the bombarding ions, the results forecast deepening of the notch at all lines.

\section{SIDEWALL PROFILE EVOLUTION RESULTS}

Profile evolution simulations for the structure of Fig. 1 have been performed as described elsewhere, ${ }^{2}$ and the profiles obtained for a fixed number of ions ${ }^{15}$ entering each trench are shown in Fig. 6 for various poly-Si thicknesses $\left(t_{p}\right)$. We begin with the case of Fig. 6(a) corresponding to $t_{p}=0.4 \mu \mathrm{m}$. Notching occurs already everywhere but is most clearly discernible at the inner side of the outermost lines ("classic" notching). Increasing the mask thickness [Fig. 6(b) and (c)] worsens notching at all lines as anticipated earlier. The profile of Fig. 6(c) for $t_{p}=1.1 \mu \mathrm{m}$ exhibits the broadest and deepest notch at the edge line, a consequence of the increased flux and energy of ions deflected towards the poly-Si sidewall. Notice that notching has also affected significantly both poly-Si sidewalls (B-left and B-right) of the neighboring line, despite the absence of electrical connection to the edge line. The extent of notching at each line corresponds perfectly to the characteristics of the nearby potential maximum (see Figs. 2 and 3).
The trends in notching at the various lines are quantified in Fig. 7, where the absolute notch depth for a fixed (but arbitrary) overetching time is plotted as a function of $\phi$ (or poly-Si thickness at the top axis). It is interesting to note that notching of the B-right sidewall is already apparent before the B-left sidewall is affected. The differences are more readily discernible as $\phi$ gets larger. Thus, increasing the thickness of the gate electrode-to reduce oxide degradation problems due to charging-is predicted to lead to more severe notching at all lines. In the case of extremely thin masks, the notches at the intermediate lines are expected to look very similar to that of the edge line (i.e., deeper and broader).

\section{SUMMARY AND CONCLUSIONS}

Detailed simulations of pattern-dependent charging and profile evolution during the overetching of transistor gate electrodes show that the angle of electron irradiance of the conductive part of the sidewalls is an important parameter for charging damage. When this angle is increased by increasing the gate electrode thickness (at constant mask thickness), a larger electron flux to intermediate gate electrodes contributes to the reduction of their charging potentials. Lower potentials imply a decrease in tunneling currents through the underlying gate oxide with a concomitant reduction in oxide degradation and breakdown. Simultaneously, the reduced sidewall potentials perturb the local ion dynamics so that the flux and energy of ions-deflected towards the sidewalls-increases. Notching worsens and, for large values of the electron irradiance angle, it affects all lines in a lineand-space structure. Since notching can be reduced independently by changing the overetch chemistry, ${ }^{2}$ there appears to be a benefit in the combination of a thinner mask with thicker gate electrode. However, this benefit must be weighed carefully against etch selectivity and throughput requirements, which will be affected by any changes in mask and gate electrode thickness, respectively.

Note added in proof: Fujiwara and co-workers [N. Fujiwara, T. Maruyama, S. Ogino, and M. Yoneda, Jpn. J. Appl. Phys. 36, 2502 (1997)] reported recent observations of notching at intermediate lines (not electrically connected) during etching of $\mathrm{WSi}_{2}$ in high-density $\mathrm{Cl}_{2}$ plasma; thick photoresist $(1.1 \mu \mathrm{m})$ was used as the mask, while the polycide was only $0.3 \mu \mathrm{m}$. Etching of similar poly-Si structures under the same conditions did not cause notching at intermediate lines. The authors attributed the difference in behavior to heavy metal deposition on the photoresist sidewalls during the polycide etch. Once a conductive film forms, it provides a path to more plasma electrons-which would normally charge the insulating photoresist sidewalls-to reach the polycide gate; there, "they recombine with ions to increase notching." Although the role of the gate potential and its influence on local ion dynamics was not understood, the explanation offered is remarkably accurate. The conductive sidewall deposit is exactly equivalent to having longer gate sidewalls, with all the consequences discussed in the present article. 
Further, for a fixed total (mask+gate) aspect ratio of a polycide and poly-Si structure, the conductive sidewall film in the polycide case implies a thinner insulating mask, which worsens notching at the inner side of the outermost line; this experimental observation validates simulation predictions of notching when using thinner masks, discussed in a Rapid Communication appearing in this issue [G. S. Hwang and K. P. Giapis, J. Vac. Sci. Technol. B 15, xxx (1997)].

\section{ACKNOWLEDGMENT}

This work was partially supported by a NSF Career Award to K.P.G. (CTS-9623450).

${ }^{1}$ T. Nozawa, T. Kinoshita, T. Nishizuka, A. Narai, T. Inoue, and A. Nakaue, Jpn. J. Appl. Phys. 34, 2107 (1995).

${ }^{2}$ G. S. Hwang and K. P. Giapis, J. Vac. Sci. Technol. B 15, 70 (1997).

${ }^{3}$ K. Hashimoto, Jpn. J. Appl. Phys. 32, 6109 (1993); 33, 6013 (1994).

${ }^{4}$ G. S. Hwang and K. P. Giapis, J. Appl. Phys. 89, 566 (1997)..

${ }^{5}$ M. A. Lieberman and A. J. Lichtenberg, Principles of Plasma Discharges and Materials Processing (Wiley, New York, 1994).

${ }^{6}$ R. Kraft, T. Boonstra, and S. Prengle, J. Vac. Sci. Technol. B 14, 543 (1996).
${ }^{7}$ C. C. Cheng, K. V. Guinn, V. M. Donnelly, and P. I. Herman, J. Vac. Sci. Technol. A 12, 2630 (1994).

${ }^{8}$ G. S. Hwang and K. P. Giapis, J. Appl. Phys. 89, 572 (1997).

${ }^{9}$ The angle $\phi$ can also be increased by changing the device rule, i.e., by increasing the trench width for a given feature design; in this case, the aspect ratio decreases. As the features get separated by larger distances, both poly-Si charging potentials and notching decrease. Thus, the trend for the line potentials still holds but that is not the case for notching. We have not yet discovered what combination of parameters will describe the notching trends.

${ }^{10}$ The assumption of complete dissociation in the chlorine plasma merely implies that no $\mathrm{Cl}_{2}^{+}$is considered in the calculation. Unless it is the dominant ion, the significance of $\mathrm{Cl}_{2}^{+}$is reduced by its larger translational energy threshold for reaction than that for $\mathrm{Cl}^{+}$; see Ref. 2 .

${ }^{11}$ Tunneling currents through the thick gate oxide are disregarded; thus, the present study focuses on a worst-case scenario, aiming to reveal where breakdown is most likely to occur from excessive charging.

${ }^{12}$ G. S. Hwang and K. P. Giapis, Appl. Phys. Lett. 70, 2377 (1997).

${ }^{13}$ Although small, contributions from the ion angular distribution (full width at half-maximum of $8.6^{\circ}$, see Ref. 2) exist and are accounted for.

${ }^{14}$ This conjecture is based on maintaining the substrate potential close to 0 $\mathrm{V}$.

${ }^{15}$ No overetching time is stated because it is impossible to calibrate the reactive ion flux without an etching experiment of a structure similar to the simulated one. 